\title{
Erratum to: Takeoff Monitoring Algorithm with Prediction
}

V. I. Garkushenko ${ }^{a, *}$ and P. A. Lazareva ${ }^{a}$

${ }^{a}$ Tupolev Kazan National Research Technical University, ul. Karla Marksa 10, Kazan, 420111 Tatarstan, Russia *e-mail:vigark@yandex.ru

Received November 9, 2020

DOI: $10.3103 / \mathrm{S} 1068799820030289$

The TeX presentation of formulas was updated in HTML file.

The original article can be found online at https://doi.org/10.3103/S1068799820020063. 